
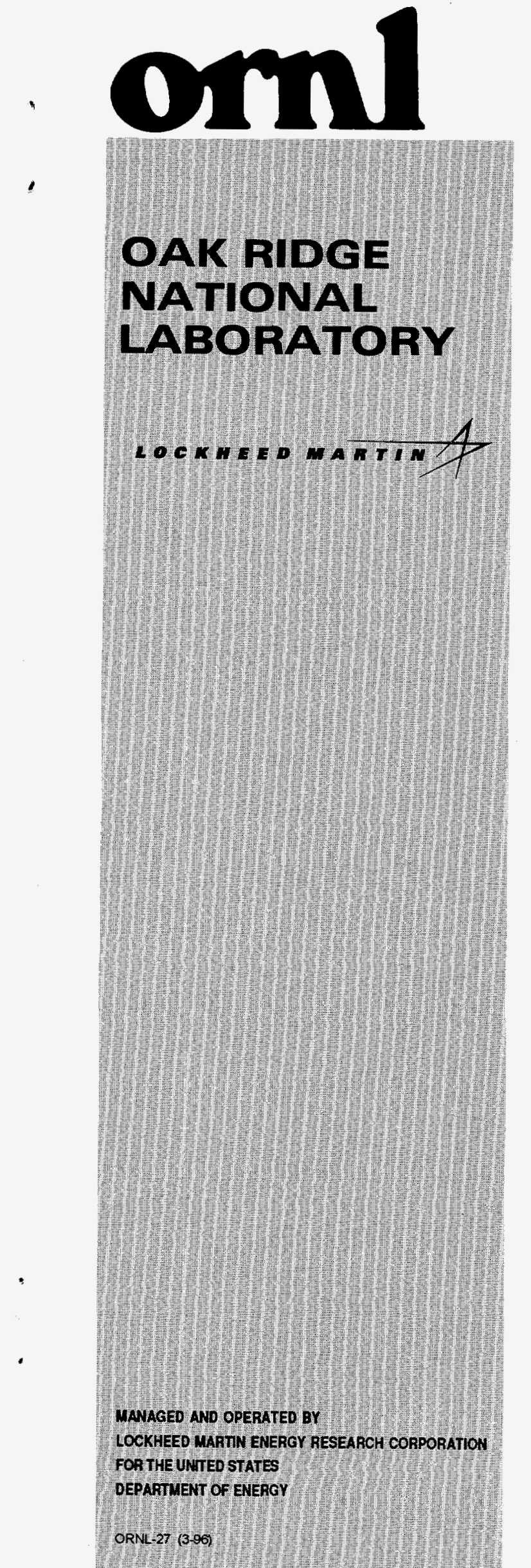

NANAGED AND OPERATED BY

LOCKHEED MARTN ENERGY RESEARCH CORPORATION FOR THE UNTEO STATES

OEPARTIUENT OF ENERGY
ORNL/TM-13619

\title{
Causal Models of Trip Replanning in TravTek
}

\author{
J. C. Schryver
}

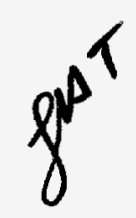

MASTER 
This report has been reproduced directly from the best available copy.

Available to DOE and DOE contractors from the Office of Scientific and Technical Information, P.O. Box 62, Oak Ridge, TN 37831 ; prices available from (423) 576-8401, FTS 626-8401.

This report was prepared as an account of work sponsored by an agency of the United States Government. Neither the United States Government nor any agency thereof, nor any of their employees, makes any warranty, express or implied, or assumes any legal liability or responsibility for the accuracy, completeness, or usefuiness of any information, apparatus, product, or process disclosed, or represents that its use would not infringe privately owned rights. Reference herein to any specific commercial product, process, or service by trade name, trademark, manufacturer, or otherwise, does not necessarily constitute or imply its endorsement, recommendation, or favoring by the United States Government or any agency thereot. The views and opinions of authors expressed herein do not necessarily state or reflect those of the United States Government or any agency thereot. 


\section{DISCLAIMER}

Portions of this document may be illegible electronic image products. Images are produced from the best available original document. 
ORNL/TM-13619

\title{
Causal Models of Trip Replanning in TravTek
}

\author{
Jack C. Schryver \\ Computer Science and Mathematics Division
}

Date Published: July 1998

Research sponsored by the

Laboratory Directed Research and Development Program

of the

OAK RIDGE NATIONAL LABORATORY

managed by

LOCKHEED MARTIN ENERGY RESEARCH CORP.

for the

U.S. DEPARTMENT OF ENERGY

under contract DE-AC05-96OR22464 

TABLE OF CONTENTS

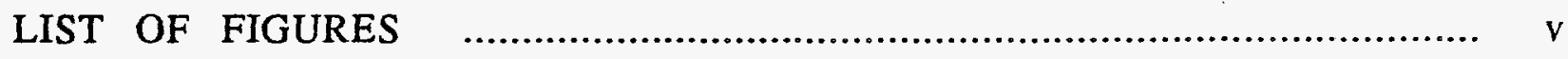

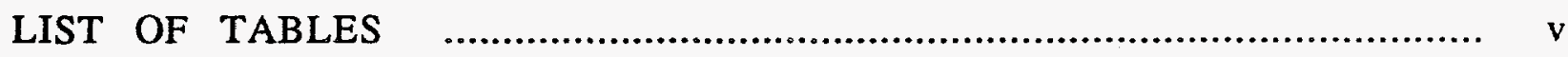

ABSTRACT

1. INTRODUCTION TO THE TRAVTEK OPERATIONAL FIELD TEST

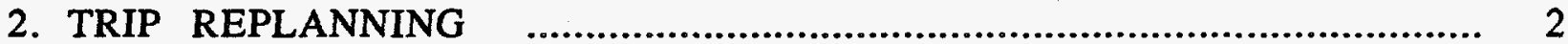

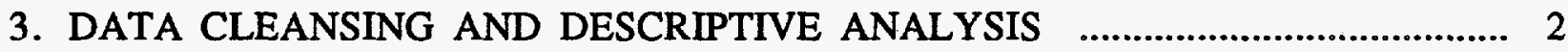

4. CAUSAL ANALYSIS

5. DISCUSSION

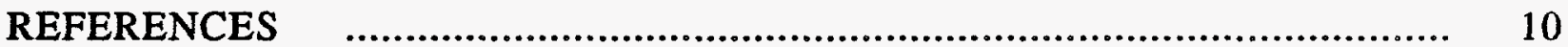




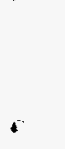




\section{LIST OF FIGURES}

Figure 1. A causal network of replanning variables ........................................... 5

Figure 2. Causal network with path coefficients …................................................... 7

\section{LIST OF TABLES}

Table 1. Mean Statistics for TravTek Variables …................................................... 4

Table 2. Replanning Correlation Matrix f........................................................... 6 
i 


\begin{abstract}
The TravTek operational field test was conducted to evaluate the effectiveness of route planning, route guidance and various navigational aiding modalities for Advanced Traveler Information Systems in ground vehicles. A causal network was constructed in order to achieve a better understanding of the dependencies among variables implicated in the replanning process. Causal inferences were modeled using path analysis techniques. The original Yoked Driver study reported that addition of real-time navigation planning did not increase trip efficiency during initial trip planning. Data mining of the relatively complete database revealed that the incidence of dynamic trip replanning was only $0.51 \%$ or 1 out of every 198 trips. Nevertheless, the replanning acceptance rate was $92.8 \%$, suggesting that less conservative criteria might have been acceptable to drivers. Several points can be made based upon the path analysis techniques. Drivers who rejected better route offers were more likely to be male renters; rejected routes were apparently offered at earlier times with a lower predicted time savings and fewer maneuvers. Failure to accept a better route also apparently resulted in fewer wrong-turn deviations. Contrary to expectations, wrong-turn count and time loss appeared as semi-independent "hubs" in the resultant causal network. Implications of the path analysis are discussed. Proposals for in-vehicle information systems are formulated to increase driver participation as co-planner, and increase the likelihood that trip replanning will positively impact trip efficiency.
\end{abstract}




\subsection{Introduction to the TravTek Operational Field Test}

Intelligent Transportation Systems (ITS) have been under development during the past decade for the purpose of enhancing surface vehicle safety through computer-based automotive and commercial vehicle information and control systems. The U. S. Department of Transportation has conducted a series of operational field tests involving a type of computer-based driver aid known as an Advanced Traveler Information System (ATIS) or In-Vehicle Information System. TravTek was an early operational field test completed in 1994. The TravTek study captured multi-gigabytes of vehicle and driver data testing vehicles equipped with ATIS systems in the metro Orlando area.

An important objective of the TravTek study was to evaluate the effectiveness of route planning, route guidance and various navigational aiding modalities. Three experimental conditions provided a services-only configuration, the Navigation configuration which included services, route planning and route navigation, and a "Navigation Plus" (NP) configuration incorporating functionality of the other two conditions with addition of real-time data communicated by a Traffic Management Center. Real-time data arrived every minute to the on-board navigation computer, and included current travel link times and incident information which the navigation computer could use to calculate new travel route times. The NP-configured system received broadcasts for historical link times as functions of time of day and day of week, link times calculated from roadway loop detectors, link times based on probe reports from other TravTek vehicles, police reports, and city maintenance reports and road closures. The NP system used this information to continuously evaluate alternative routes due to changing traffic conditions. If an alternative route presented a faster travel time while on-route, the NP system could offer a better route based on real-time information to the traveler for approval. The only other occasion for offering a route to the traveler once a trip had begun was when the vehicle had taken a wrong turn and gotten offroute. The system notified the traveler of the off-route status, and calculated a route back to the original route. The traveler could not request an alternative or new route on their own initiative.

Seventy-five of the one hundred cars used in the operational field test were used by 4,354 Orlando visitors driving rental cars for relatively short periods. Navigation Plus rentals averaged 5.2 days. The remaining cars were used by 53 local residents for 6 to 8 weeks. Local drivers tended to use the cars for longer time intervals than the renters.

The TravTek global evaluation reported that route planning saved travelers planning time, and that the route guidance system uniformly reduced travel time (Inman and Peters, 1996). Other evaluations have focused on the effectiveness of different modes of route guidance (e.g., Dingus, et al., 1994). But what about the benefit of real-time route planning? One of the nine individual TravTek studies, the relatively small (but controlled) Yoked Driver study was designed to help answer this question. Three vehicles were yoked such that they departed from the same origin for the same destination at about the same time. Yoked trips occurred during evening rush hour through the congested downtown area. One vehicle in each triplet was configured to either the service, navigation or NP condition. Comparisons of navigation and NP conditions revealed that real-time information did not significantly reduce travel time. The Yoked Driver evaluation study (Inman, Sanchez et al., 1996) explained this finding by suggesting that the navigation system was insensitive to real differences between alternative routes due to inaccurate estimates of (fluctuation in) travel time for arterials. Nevertheless, different routes were planned in 50 out of 67 cases on the basis of the availability of real-time traffic information. NP-configured vehicles took significantly longer routes on lower class roadways but completed them in the same time as navigation-configured vehicles. The investigators concluded that using real-time information to avoid congestion in NP-configured vehicles could indirectly aid non-TravTek users, who presumably benefit from relief brought about by a more even distribution of vehicles throughout 
the traffic grid. The conclusion was supported by the marginally significant finding of less congestion in NP-configured vehicles as compared to the other configurations.

If TravTek users did not directly benefit from real-time information at the initiation of trip planning, did they benefit from the availability of dynamic trip replanning? Replanning was an option for all users of the NP-configured system, which could respond to sudden onset of traffic delays caused by unplanned incidents, such as traffic accidents. These unplanned traffic events might not be detected during initial trip planning, because they are transient phenomena emerging from the dynamics of traffic flow.

\subsection{Trip Replanning}

The planned evaluations of TravTek did not include a comprehensive statistical analysis of trip replanning for NP-configured vehicles in the larger rental and local user studies. One reason for this omission might have been the lack of extensive trip data on replanning occurrences. Reanalysis of the entire TravTek database shows that initiation of trip replanning was reported in only 110 trips out of a possible 21,752 moving trips where a NP-configured system installed an initial route $(0.51 \%)$ according to queries of the TravTek database. This figure includes data only from rental, local, special and yoked study users. The reported percentage of usage is equivalent to replanning activity occurring on 1 out of every 198 trips.

The precise reasons for such a low replanning percentage are not exactly clear. Certainly some of the TravTek NP system trips were short enough to limit the possibility of replanning, and still other trips terminated prematurely before reaching the destination. Although it was not indicated in the evaluation studies, it is possible that some travelers used the navigation system for initial route planning, and because they did not desire to use the route guidance feature, subsequently turned off the navigation system for the trip duration. Perhaps the number of viable alternative routes for many common trips in the Orlando area were substantially limited due to highway network configuration. Another explanation is that effects of locally-initiated route incidents tended to propagate rapidly throughout the grid, quickly settling into a new steady state characterized by an even geographic distribution of traffic density. One consequence of this simple traffic flow model is that all delays affect the traffic grid equally, eliminating any relative advantage among alternative routes. Finally, the navigation system may have employed overly conservative criteria in offering better routes to drivers. We shall return to this explanation in the concluding remarks.

Although we cannot directly determine whether replanning actually saved travel time in TravTek vehicles, Inman, et al. (1996) reported that renters who accepted better routes rated the TravTek system in general similarly to other NP system users, but that they were more convinced that the navigation system helped them to save time in reaching their destinations as compared to other NP system users. Despite the low incidence of navigation system-initiated replanning, we may reasonably wish to estimate the effectiveness of trips replanned by the system, and the contributions of important causal variables related to replanning and their effects on driving behavior.

\subsection{Data Cleansing and Descriptive Analysis}

Trip data from 111 replanning episodes representing 92 distinct trips were selected from a 27 GB TravTek database. The renter and local user study reported only 42 instances of better routes being offered to the driver. The present analysis includes at least 27 episodes involving special vehicles reserved for AAA, GM, Florida DOT, and FHWA, as well as 5 episodes from the Yoked Driver 
Study. However, this still leaves a discrepancy of 37 replanning episodes in rental and local-user vehicles. It is concluded that the present collection represents a more complete sample of the total number of replanning episodes in the entire TravTek study as compared to the planned evaluations. The data were originally stored in multiple FoxPro database tables and archived on $8 \mathrm{~mm}$ tapes. These data were restored and converted first to flat files and subsequently to relational tables in an Oracle database stored on a Unix workstation. A series of SQLPLUS queries were posed to extract critical variables such as the proposed time of the better route, estimated time of the present route, duration of the entire trip, estimated number of maneuvers, and number of off-route warnings delivered by the ATIS interface. The Oracle database was also queried to obtain driver information such as gender, renter/local status, and age. A Pro C program was written to perform the required queries of the TravTek database in order to determine the acceptance latency after a better route was offered the driver.

Some of the critical derived tables were cleaned, and several duplicate records removed from the analyses. Inconsistencies were noted with the "proposed time" and "route distance" variables in the TravTek database. The inconsistencies vanished when the values for these two variables were exchanged for all but 7 records. Complete data was not obtained for most variables used in the statistical analyses. For example, the numbers of records obtained for age, sex, renter status, acceptance latency and number of off-route warnings were respectively $76,81,77,56$, and 64 out of a possible 111 records.

Means for the major replanning variables are given in Table 1. The first column refers to all episodes where the system offered the driver a better route. The second column, which is a proper subset of the first column, considers only those better route offers which were never accepted by the driver. An offer was marked as not accepted if an acceptance event did not occur between the time of the offer and arrival at the destination or trip termination, or if another offer was made before the driver responded to the first offer. The third column lists database-wide estimates of the same replanning variables.

Clock time (at replanning) was measured in seconds relative to trip start. Elapsed time intervals (present route time, proposed time, actual time) were measured from the time a better route was offered to the time of destination arrival, even if another replanning episode occurred within this broad interval. Count variables refer to events occurring within the actual elapsed time interval. The acceptance latency is the interval from the time a better route was offered to the event labelled "better route accepted."

Proposed time savings and time loss were calculated log-ratios. Proposed time savings is the natural logarithm of the ratio of proposed time to present route time. Time loss is the natural logarithm of the ratio of actual time to proposed time. Log transforms were applied to these two variables for two reasons. First, a few extreme outliers were discovered in the proposed time estimates. At least in the case of time savings, the average of the ratio would be highly sensitive to outliers. Second, averages of ratios are more sensitive to values containing larger numerators than larger denominators. The log-ratios have simple and direct interpretations. A positive log-ratio proposed time savings is associated with a positive time savings estimate, and negative ratios refer to lack of time savings. A positive log-ratio time loss denotes positive lost time, whereas a time savings would accrue in a negative log-ratio time loss.

Database-wide samples were large enough that database means were taken as population values for purposes of statistical inference. Simple z-tests were performed on column 1 means for age, sex, renter status, time at (re)planning, maneuver count, and wrong-turn count calculated from the replanning episodes (first column) to verify the null hypothesis that the samples were derived from 
Table 1. Mean Statistics for TravTek Variables (SD in parentheses)

\begin{tabular}{||l|rr|r|r|}
\hline Variable & Replanned (all) & $\begin{array}{l}\text { Replanned } \\
\text { (not accepted) }\end{array}$ & $\begin{array}{c}\text { TravTek } \\
\text { database }\end{array}$ \\
\hline Age (yrs.) & 42.7 & $(1.324)$ & 42.8 & 43.8 \\
\hline Sex (1=male) & 0.79 & $(0.045)$ & 1.00 & 0.56 \\
\hline Renter status (1=renter) & 0.662 & $(0.054)$ & 0.833 & 0.888 \\
\hline Time at replanning (sec.) & 1265.28 & $(98.1)$ & 781.08 & 929.79 \\
\hline Present route time (est.) & 103108 & $(41636)$ & 2075.25 & 6376.49 \\
\hline Proposed time (est.) & 889.72 & $(54.03)$ & 1202.5 & 6417.9 \\
\hline Actual time to destination (sec.) & 2454.83 & $(449.0)$ & 2396.68 & $* * *$ \\
\hline Maneuver count & 9.87 & $(0.39)$ & 7.13 & 6.66 \\
\hline Acceptance latency (sec.) & \multicolumn{1}{|c|}{11.28} & $* * *$ & $* * *$ \\
\hline Wrong-turn count & 1.875 & $(0.265)$ & 1.375 & 0.630 \\
\hline Proposed time savings (rel) & 0.665 & $(0.04)$ & 0.594 & $* * *$ \\
\hline Time loss (rel) & 0.804 & $(0.083)$ & 0.807 & $* * *$ \\
\hline
\end{tabular}

the database-wide population (third column). Drivers involved with replanning episodes were significantly more likely to be male $(\mathrm{z}=5.11 ; \mathrm{p}<.001)$ and local $(\mathrm{z}=4.19 ; \mathrm{p}<.001)$ as compared to drivers in the entire TravTek study. Age was not a significant factor in determining whether a better route would be offered. Drivers could not request replanning; it is suggested that perhaps local males are more likely to select origin/destination pairs that are amenable to replanning. Replanning episodes occurred at a significantly later time during the trip, yet were associated with significantly more maneuvers $(\mathrm{z}=8.23 ; \mathrm{p}<.001)$ and resulted in a significantly greater number of off-route excursions $(\mathrm{z}=4.70 ; \mathrm{p}<.001)$ as compared to the general planning episode. This is not unexpected given the fact that replanned routes were not initially selected by the navigation system during trip planning.

Further analysis showed that 8 of 111 confirmed offers for better routes were not accepted - this figure is equivalent to a $92.8 \%$ acceptance rate. Three of the eight failures to accept could be attributed to a single driver/trip. A very high acceptance rate indicates that replanned routes, when offered, were nearly always accepted by drivers. The group means provided in column 2 of Table 1 suggest that drivers who failed to accept better routes offered by the system were even more likely to be male renters as compared to the entire sample of drivers who were offered better routes. Better routes that were not accepted were apparently offered at earlier times with lower predicted time savings and fewer maneuvers. A failure to accept also apparently resulted in fewer wrong-turn deviations. These trends suggest that the decision to reject a better route offer was usually a sound one. These trends could not be confirmed by statistical analysis, due to the very small number of cases where better offers were not accepted. Inman et al. (1996) reported that the Voice Guide was turned off on many instances of failure to accept a better route, whereas the Voice Guide was turned on when better routes were accepted. There was also some evidence drivers who failed to accept better routes did not understand the system sufficiently well. The implication was that if travelers were aware that a better route was offered, they accepted the offered route. 


\subsection{Causal Analysis}

One approach to a better understanding of the global structure of the replanning process is to portray the major causal relationships in a single diagram. We might construct a causal network composed of several variables in order to model dependencies among variables associated with the replanning process. A causal network consists of a set of nodes that represent the dependent variables, and directional links connecting node pairs. Some node pairs are connected directly by a causal link, whereas other variable pairs are connected only indirectly through one or more causal pathways. Using a method known as path analysis (Blalock, 1979), we can model causal inferences regarding the replanning variables. Path analysis is a mathematical technique that has frequently been used to model causal networks composed of continuous variables. One might think of path analysis as a continuous variable analogue of Bayesian Belief Networks, which are mostly used to model dependencies among nominal variables.

The beta coefficient (directional link) for a given independent/dependent variable pair in a causal network is computed by taking the standardized beta weight for the associated independent variable in a multiple regression on the dependent variable. Beta coefficients for the entire path diagram are obtained by setting up a set of linear recursive equations beginning with the main dependent variable (i.e., time loss) (Wermuth, 1980.) Additional multiple regressions are performed in recursive fashion until all variables in the network are included in the analysis.

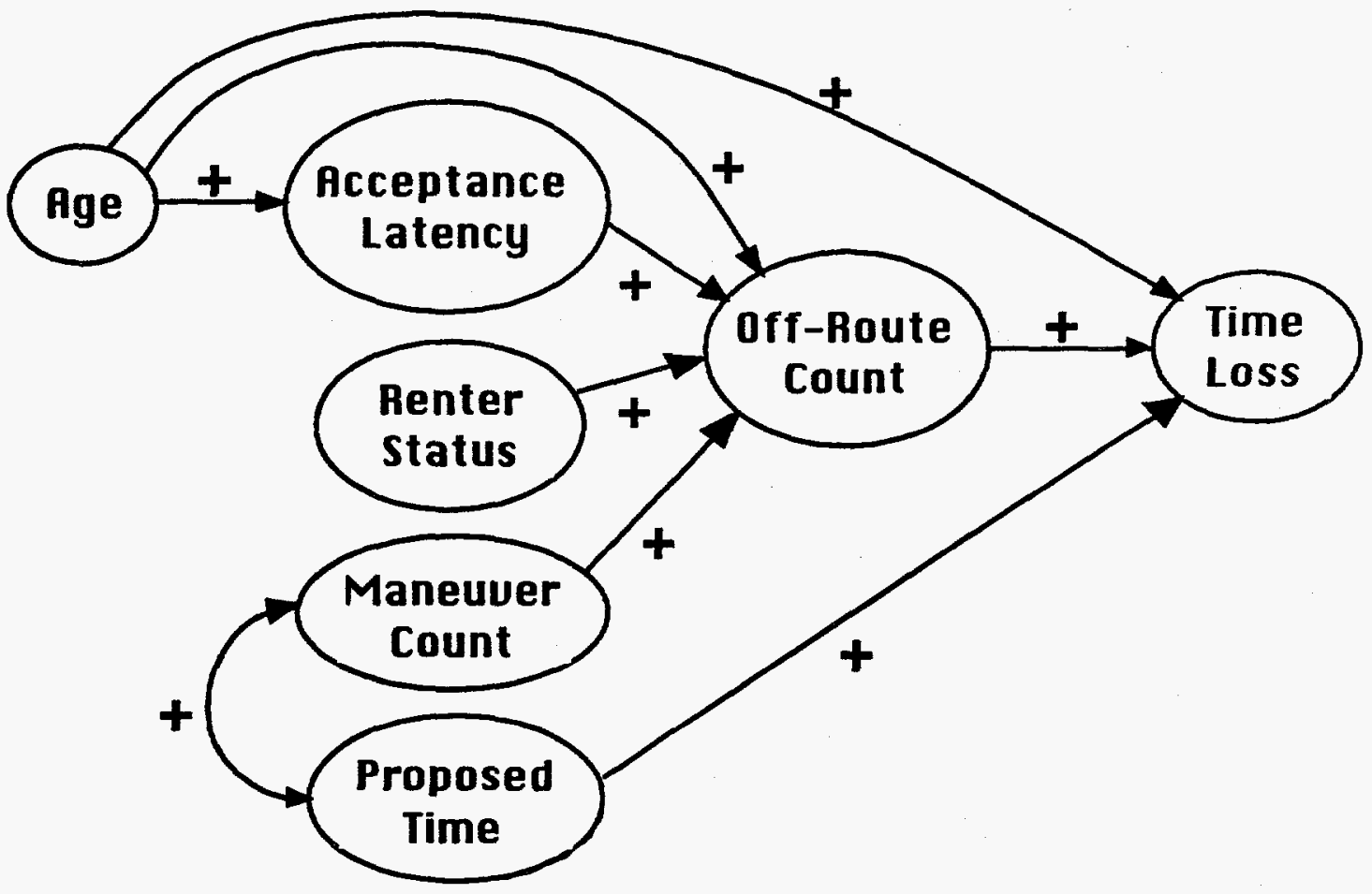

Figure 1. A causal network of replanning variables.

Path analysis is based on the correlation matrix, and correlations do not convey information about causality. However, it is partially possible to test for causal links by examining the partial correlations in a network, assuming that all important variables are modeled. In the simple causal model depicted by: A $\rightarrow>$ B $-->$ C, the variable A causes B, and B causes C. All possible 
correlations are positive, but if the model is correct the partial correlation between $\mathrm{A}$ and $\mathrm{C}$, while controlling for B should equal 0 .

For example, Figure 1 shows a possible network based on prior expectations. In this model, wrong-turn count is the main direct factor in predicting time loss. Other variables associated with time loss (acceptance latency, renter status, maneuver count) exert only indirect effects through wrong-turn count. Acceptance latency is linked to wrong-turn count because it is hypothesized that large latencies increase the probability of missing an initial maneuver along a new route.

The correlation matrix for the variables shown in Figure 1 appears in Table 2. Renter status and sex, which are normally treated as bivalued nominal values, were recoded as numerical variables with discrete values $(0=$ female, $1=$ male; $0=$ local, $1=$ renter $)$ for consistency in all further analyses.

Table 2. Replanning correlation matrix (positive values in bold.)

\begin{tabular}{||r|r|r|r|r|r|r|r|r||}
\hline \multicolumn{1}{|c|}{ Variable } & Age & \multicolumn{1}{c|}{ Sex } & $\begin{array}{c}\text { Renter } \\
\text { status }\end{array}$ & $\begin{array}{c}\text { Prop. } \\
\text { time }\end{array}$ & $\begin{array}{c}\text { Maneuver } \\
\text { count }\end{array}$ & $\begin{array}{c}\text { Acceptance } \\
\text { latency }\end{array}$ & $\begin{array}{c}\text { Wrong- } \\
\text { turn count }\end{array}$ & $\begin{array}{c}\text { Time } \\
\text { loss }\end{array}$ \\
\hline Age & 1.000 & -.194 & $\mathbf{+ . 2 7 2}$ & 0.000 & -.095 & +.135 & -.306 & -.093 \\
\hline Sex & -.194 & 1.090 & -.200 & -.150 & +.088 & -.044 & +.067 & +.198 \\
\hline Renter status & +.272 & -.200 & 1.000 & +.318 & -.380 & -.023 & +.288 & -.250 \\
\hline $\begin{array}{l}\text { Proposed } \\
\text { time }\end{array}$ & -0.000 & -.150 & +.318 & 1.000 & -.103 & +.183 & -.005 & -.562 \\
\hline $\begin{array}{l}\text { Maneuver } \\
\text { count }\end{array}$ & -.095 & +.088 & -.380 & -.103 & 1.000 & -.070 & +.265 & +.326 \\
\hline $\begin{array}{l}\text { Acceptance } \\
\text { latency }\end{array}$ & +.135 & -.044 & -.023 & +.183 & -.070 & 1.000 & -.099 & -.148 \\
\hline $\begin{array}{l}\text { Wrong-turn } \\
\text { count }\end{array}$ & -.306 & +.067 & +.288 & -.005 & +.265 & -.099 & 1.000 & +.096 \\
\hline Time loss & -.093 & +.198 & -.250 & -.562 & +.326 & -.148 & +.096 & 1.000 \\
\hline
\end{tabular}

Careful inspection of the correlation matrix suggested a different causal network as shown in Figure 2. Black lines denotes positive links, and gray lines show the negative links. Line thickness conveys the strength of the link, or the beta coefficient. The latent structure depicted in Figure 2 should be evaluated in tentative terms, since the sample size was too small to permit meaningful significance testing.

The causal network depicted in Figure 2 was constructed according to the following procedure. A multiple regression was first performed on time loss with maneuver count, proposed time and sex as predictor variables. The only linear recursive equation to follow from time loss was proposed time, which was regressed onto renter status. Wrong-turn count was a secondary dependent variable. Wrong-turn count was regressed on maneuver count, renter status and age. On a second level regression analysis, renter status was regressed onto sex. Finally, acceptance latency is regressed onto proposed time. The causal network contains no loops, as required by path-analytic methods. 
Renter status was moderately correlated with most other replanning variables, and is reflected by its central position in the causal network. However, inspection of Figure 1 yields no direct link between renter status and time loss, although the correlation is -.250 . Partial correlations between these variables, when separately controlling for effects of maneuver count and proposed time respectively, are reduced to -.144 and -.091 . It was concluded that the causal pathways from renter status through maneuver count and proposed time to time loss was sufficient to account for the relationship between renter status and time loss.

Indirect effects can be estimated by summing the magnitudes of all pathways from one variable to another. For example, if the beta coefficient for $\mathrm{A}->\mathrm{B}$ is 0.6 , and the beta coefficient for the path from $B \rightarrow C$ is 0.5 , then the magnitude of the causal relationship $A \rightarrow C$ is $(0.5)^{*}(0.6)=0.3$. Indirect effects of renter status on time loss total to $(-.380) *(.163)+(.318) *(-.563)$ or -.241 . However, partial correlations between renter status and wrong-turn count controlling individually for maneuver count and age increased the simple correlation from +.288 to +.436 and +.405 respectively. Therefore, a direct link between renter status and wrong-turn count was retained in the causal network. The partial correlation between age and wrong-turn count controlling for renter status was also moderately extreme $(-.417)$, which justified retention of that link in the causal network.

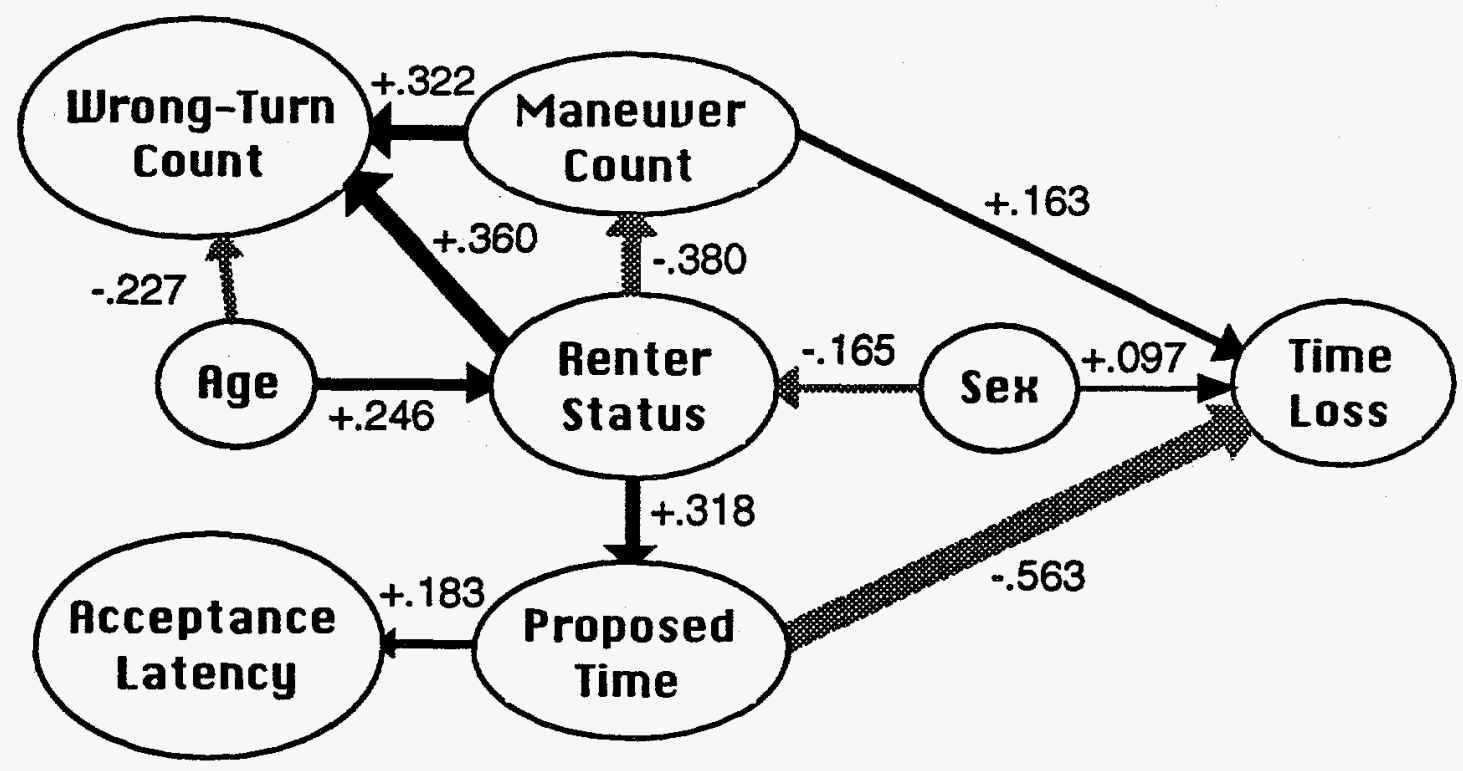

Figure 2. Causal network with path coefficients.

Proposed time, maneuver count and sex were the only direct associations into time loss (the primary dependent variable.) The multiple regression equation estimated from the data is:

Time Loss $=-0.000868 \times$ Proposed Time $+0.0371 \times$ Maneuver Count $+0.213 \times$ Sex

The resulting positive link between maneuver count and time loss was expected, and is consistent with a trip timing model where each distinct maneuver increments cumulative time loss by a constant proportion. Males lost slightly more time per trip than females. The beta coefficient connecting proposed time with time loss $(-.563)$ was the most extreme in the entire network. This result is not surprising from a purely mathematical perspective, because the predictor variable 
(proposed time) was expressed in the denominator of the predicted variable (time loss). We can estimate the proposed time required to generate an equal length actual trip time by inserting the sample means for sex and maneuver count into the regression equation for time loss. By solving for proposed time by setting time loss equal to zero (i.e., actual time = proposed time), we find that proposed trips shorter than 1782 seconds tended to be underestimated, but overestimated when proposed trips times exceeded 1782 seconds. The average proposed route time was 892 seconds; a majority of trips were therefore likely to be underestimated.

The Yoked Driver study indicated that wrong turns in vehicles with navigation guidance tended to be errors of commission (i.e., a turn at a wrong place or wrong direction), and that control group vehicles were associated with errors of omission, (i.e., the vehicles continued when a maneuver was planned.) Wrong-turn count was expected to be a central mediating link to time loss in the network. However, wrong-turn count and time loss were essentially uncorrelated, forming two distinct "hubs" or subnetworks in the overall network. Maneuver count was the only variable directly linked to both wrong-turn count and time loss subnetworks. This causal pattern suggests that perhaps it is too simplistic to regard wrong-turn count only as a direct measure of performance deficit. Indeed, getting off-route may occasionally signify a deliberate attempt to beat the route plotted by the system by trying a slightly different route. Even when getting off-route turns out to be non-optimal or accidental, the traveler may often recover gracefully by planning and executing a return route back to the system-initiated route without substantial time loss. Indeed, active behavior such as this may even enhance traveler familiarity with the traffic network and facilitate the development of a "mental map" of the area. It would be expected that this explanation is more likely to be accurate for local travelers as compared to renters who are less familiar with the terrain. This line of thought evokes a traveler/system control model in which both traveler and navigation system are co-planners in real-time, instead of a more hierarchical control model in which the navigation system is the active planner, and the traveler passively implements navigation system plans.

Wrong-turn count was positively correlated with maneuver count. This association is intuitive, because more opportunities for wrong turns will almost inevitably lead to more wrong turns. The increased vulnerability to getting lost is a major reason to favor trips with fewer maneuvers. Renters committed more wrong turns than local drivers, which would be expected due to the greater familiarity of local drivers with the area and the arterial network.

Dingus et al. (1997) found that the cognitive workload increment imposed by the navigation system on older drivers resulted in slower and more cautious driving. The conservativeness of older drivers is consistent with the negative correlation between age and wrong-turn count, even though older drivers might be expected to commit more navigation errors. However, an apparent greater determination of older drivers to remain on-route may have outweighed a possible greater likelihood to commit driving errors.

Acceptance latency is a mini-hub in the causal network that has proposed time as its only predictor variable. Longer replanned routes required more time to accept, which may be attributable to extended decision-making time. Longer routes may require more time to evaluate than shorter routes. This link in the causal network suggests that drivers actively evaluated each alternative route offered by the navigation system, and did not simply accept blindly whatever was offered (as the high acceptance rate might suggest.) It was expected that acceptance latency would increase the number of wrong-turns, but there was no evidence for a direct link in the network diagram. A long acceptance latency might have increased the likelihood of missing the first turn on the new route, but evidently most acceptance latencies occurred prior to reaching the next turn along the new route. 


\subsection{Discussion}

The TravTek replanning data suggest that when trip replanning was used, it was accepted by travelers and provided effective navigation aiding. It is almost axiomatic that improvement of dynamic estimates of link travel time can lead to measurable benefits of real-time navigation in terms of trip efficiency. For example, Balke (1994) found that although speed measurements from the freeway surveillance system are able to produce consistent estimates of link travel times, the delay measurements form the computerized traffic signal system did not produce similarly consistent estimates of link travel time for the arterial street network. However, in order to validate the full benefit of real-time dynamic trip replanning, it is critical to achieve an incidence of trip replanning substantially greater than $1 / 2$ of 1 percent.

One strategy which has received little consideration in the literature is to increase traveler-system interaction by providing more information to the traveler (if desired), and encouraging greater traveler participation as co-planner (and co-replanner). A few special-purpose design features of a real-time vehicle navigation system are highlighted below as potential means of increasing replanning incidence.

- $\quad$ Allow drivers to request initiation of replanning at any time during a trip, view trip time estimates, and then accept or reject calculated routes. This option allows travelers to upgrade their navigation role beyond the level of passive acceptance.

- Provide interval estimates for direct comparison of proposed time and current trip time, or a likelihood estimate that a better route is faster, and highlight proposed routes on electronic maps. This information allows travelers to incorporate uncertainty into the decisionmaking process when considering a system-offered route.

- $\quad$ Provide post-trip feedback on estimated trip time if the old route were followed (based on dynamic post-decision link times) as well as actual trip time along the new route. These numbers provide the driver with the most accurate indication of actual route efficiency.

- Employ less conservative (or traveler-selected) criteria in offering better routes to drivers. The Navigation Plus system was programmed to offer a better route only if a significant delay was detected on the planned route, and an alternative route was discovered to considerably reduce travel time. Better routes reduced mean proposed travel time $46 \%$ over estimated mean route time, and the minimum estimated travel time reduction was $30 \%$. These figures suggest that the navigation system planned for a large cushion in deciding to offer a better route to the driver.

One issue that could not be addressed due to lack of replanning data for single drivers is the effects of experience and the influence of trust in the navigation system on acceptance of better route offers. Dingus et al. (1997) showed that drivers become familiar with the navigation system and develop strategies for more effective use of the TravTek system. Specifically, it might be expected that drivers could lose confidence in the navigation system if actual trip times based on replanning consistently exceeded proposed route time estimates, even though proposed route time estimates showed a time savings over current route estimates at the time the new route was accepted. Bonsall and Joint (1991) found that degree of acceptance declined with the quality of previous route advice. Compliance ranged from $20 \%$ to $80 \%$, where only $20 \%$ compliance was obtained when present and previous advice quality was poor. "Advice quality" was defined as a ratio of travel time on the advised route over optimal route travel time. But drivers were never able to compare actual trip times with the trip times that would have resulted if they remained on the old route. One method of boosting confidence could be to continuously update the trip time estimate of 
the old route based on real-time information, so that at destination arrival time the traveler would have a meaningful comparison of the replanned trip with other alternatives.

Of course, it would be unwise to burden the traveler with additional information if it increases cognitive workload to an extent that it interferes with primary driving tasks and compromises vehicle safety. Additional field research is required to determine the optimum level of driver involvement in the replanning process.

\section{References}

Balke, K. N. Evaluation of the quality of traffic information in the TravTek system. pp 294-299 in The Proceedings of the 1994 Annual Meeting of IVHS America, (1994).

Blalock, H. M. Jr. Social Statistics. New York: McGraw-Hill, (1979).

Bonsall, P. W. And Joint, M. Driver compliance with route guidance advice: the evidence and its implications. pp 47-56 in Vehicle Navigation and Information Systems Conference, P-253, Part 1 (October)(SAE Paper No. 912733) Warrendale, PA: Society of Automotive Engineers. Summarized in TRB/NRC Circular 419, pp 117-118, (1991).

Dingus, T. A., Hulse, M. C., McGehee, D. V., Manakkal, N., and Fleischman, R. N. Driver performance results from the camera car evaluation study. pp 1118-1122 in Proceedings of the Human Factors and Ergonomics Society 38th Annual Meeting. Santa Monica, CA: HFES, (1994).

Dingus, T. A., Hulse, M. C., Mollenhauer, M. A., Fleischman, R. N., McGehee, D. V. And Manakkal, N. Effects of age, system experience, and navigation technique on driving with an advanced traveler information system. Human Factors, 39(2), 177-199, (1997).

Inman, V. W., Fleischman, R. N. Sanchez, R. R., Porter, C. L., Thelen, L. A. and Golembiewski, G. TravTek Evaluation Rental and Local User Study. FHWA-RD-96-028, Washington, D. C.: Federal Highway Administration, (1996).

Inman, V. W. And Peters, J. I. TravTek Global Evaluation and Executive Summary, FHWA-RD96-031, Washington, D. C.: Federal Highway Administration, (1996).

Inman, V. W., Sanchez, R. R., Porter, C. L., and Bernstein, L. TravTek Evaluation Yoked Driver Study. FHWA-RD-94-139, Washington, D. C.: Federal Highway Administration, (1995).

Wermuth, N. Linear recursive equations, covariance selection, and path analysis. Journal of the American Statistical Association: Theory and Methods Section 75(372), 963-972, (1980). 


\section{INTERNAL DISTRIBUTION}

1. J. Barhen

2. R. J. Carter

3. N. W. Grady

4. H. E. Knee

5. P. F. Spelt

6-10. J. C. Schryver

11. D. R. Tufano
12. T. Zacharia

13. CSMD Reports Office

14. Laboratory Records - RC

15-16. Laboratory Records - OSTI

17. Central Research Library

\section{EXTERNAL DISTRIBUTION}

18. Bob Carroll, Office of Motor Carrier Research and Standards, Federal Highway Administration, U. S. Department of Transportation, 400 7th Street, SW, Washington, DC 20590

19. Dr. Stephen T. Elbert, Division of Mathematical, Information, and Computational Sciences U. S. Department of Energy (ER-31), 19901 Germantown Road Germantown, MD 20874-1290

20. Michael J. Goodman, Office of Crash Avoidance, National Highway Traffic Safety Administration, U. S. Department of Transportation, 4007 th St., SW, Washington, DC 20590

21. Ron Knipling, Office of Motor Carrier Research and Standards, Federal Highway Administration, U. S. Department of Transportation, 400 7th Street, SW, Washington, DC 20590

22. Joseph Peters, Intelligent Transportation Systems Joint Program Office, Federal Highway Administration, U. S. Department of Transportation, 400 7th St., SW (HVH-1), Washington, DC 20590 